\title{
EFECTOS DEL SORBATO DE POTASIO A DIFERENTES \\ CONCENTRACIONES Y TIEMPO DE EXPOSICIÓN SOBRE EL \\ CICLO CELULAR Y EL MATERIAL GENÉTICO EN MERISTEMOS \\ RADICULARES DE ALLIUM CEPA L. (CEBOLLA)
}

\section{EFFECT OF POTASSIUM SORBATE AT DIFFERENT \\ CONCENTRATIONS AND EXPOSURE TIME ON THE CELL CYCLE AND THE GENETIC MATERIAL IN ROOT MERISTEMS OF ALLIUM CEPA L "ONION"}

\author{
Joselyne Quispe, José Saldaña, Tony Verde y Shirley Valderrama \\ Universidad Nacional de Trujillo, \\ Trujillo,Perú
}

\section{RESUMEN}

Con el propósito de determinar el efecto del Sorbato de Potasio sobre el ciclo celular en meristemos radiculares de Allium cepa $L$. "cebolla" se establecieron tres grupos experimentales que se expusieron a las soluciones de Sorbato de Potasio en concentraciones de $0.5,1$ y 2 g/L; por 6,7 y 8 horas cada una y un grupo control. Luego se realizó la respectiva coloración en Orceína acética al $2 \%$ de acuerdo a la técnica de Tjio y Levan, observándose con el microscopio compuesto un total de 1500 células meristemáticas en cada uno de los tratamientos. Los resultados aplicando tratamiento después de 8 horas de exposición a la concentración de $2 \mathrm{~g} / \mathrm{L}$ mostraron una disminución del índice mitótico de $11.26 \%$, mientras que se incrementó el índice profásico en $95.57 \%$. Se produjeron también alteraciones como rupturas cromosómicas, puentes anafásicos y células binucleadas. Las pruebas y análisis de varianza, mostraron diferencias significativas entre los diferentes tratamientos, confirmado mediante la prueba de comparación múltiple de promedios de Duncan. Se evidencia un probable efecto genotóxico en el material genético por el Sorbato de Potasio y una alteración en el ciclo celular de los meristemos de A. cepa L.; así mismo, se comprueba la función de biosensor de $A$. cepa $L$. en el estudio de sustancias que puedan afectar el ciclo celular.

Palabras clave: Allium cepa, Sorbato de Potasio, Índice Mitótico, Alteración del material genético, Puentes

anafásicos, Rupturas cromosómicas, Células binucleadas.

\begin{abstract}
In order to determine the effect of Potassium Sorbate on the cell cycle in root meristems of Allium cepa L. "onion" set three experimental groups were exposed to solutions of Potassium Sorbate at concentrations of $0.5,1$ and $2 \mathrm{~g} / \mathrm{l}$, for 6,7 and 8 hours each and a control group. Later the respective color in $2 \%$ Acetic orcein according to the technique of Tjio and Levan, observed with the compound microscope a total of 1500 meristematic cells in each of the treatments. The appropriate treatment results after 8 hours of exposure to the concentration of $2 \mathrm{~g} \mathrm{/} \mathrm{L}$ showed a decrease in mitotic index of $11.26 \%$, while it increased the rate at $95.57 \%$ prophasic index. There were also alterations as chromosome breaks, anaphasic bridges and binucleated cells. The tests and analysis of variance showed significant differences between the different treatments, as confirmed by multiple comparison test of Duncan averages. It shows a probable genotoxic effect in the genetic material by Potassium Sorbate and an alteration in the cell cycle of meristems of $A$. cepa $L$., likewise, is found biosensor function of $A$. cepa $L$. in the study of substances that may affect the cell cycle.
\end{abstract}

Keywords: Allium cepa, Potassium Sorbate, Mitotic index, Abnormal genetic material, Chromosomal breaks, Anafasic bridges, Binucleated cells. 


\section{INTRODUCCION}

Actualmente, los conservantes se utilizan para proteger los productos alimentarios de la acción de los microorganismos que pueden deteriorarlos o contaminarlos. Estos conservantes pueden limitar, retardar o prevenir los procesos de la fermentación, enmohecimiento, putrefacción y otras alteraciones provocadas por bacterias $y$ hongos. Actúan bioquímicamente sobre el microorganismo, destruyendo su membrana plasmática, bloqueando su actividad enzimática o afectando a su estructura genética. Sin embargo, si bien es cierto, estos compuestos tienen efectos benéficos inmediatos para el hombre, también inducen anormalidades en las células humanas [1][2].

Uno de los conservantes más usados en la industria alimentaria, es el Sorbato de Potasio; por su alta solubilidad, efecto antibacteriano y antimicótico comprobado, por ser fácilmente catabolizado y asimilado por el organismo y por su carácter inocuo; sin embargo es irritante a las membranas [1]. Debido a sus notables propiedades químicas y físicas, es fácil de usar y no influye en el sabor ni en el olor de los productos, por lo que ha sido adoptado en muchos países como el conservante ideal para varios productos alimenticios [2][3].

El Sorbato de Potasio, detiene el crecimiento microbiano al inhibir a las deshidrogenasas involucradas en la oxidación de ácidos grasos; ocasionando la acumulación de ácidos grasos B-insaturados que son productos intermedios en el metabolismo lipídico [4] [5]. Otro mecanismo de acción esta asociado a los dobles enlaces que posee, los cuales interfieren con la actividad catalítica de las enzimas responsables del crecimiento microbiano convirtiéndose de esta manera en un eficaz fungicida [6][7].

El conservante es tipificado como "generalmente reconocido como seguro" para la salud (GRAS); sin embargo, dado su carácter artificial, probablemente interacciona químicamente con otros componentes presentes en los alimentos [8], así en la conservación de las carnes procesadas, donde es habitual el uso de sorbatos como agente antimicótico y de nitritos contra Clostridium botulinum, se ha comprobado que al difundir al interior del alimento, reacciona con los nitritos generando ácido etilnitrólico (ENA), de posible efecto cancerígeno en el hombre [8][9].
Es ese sentido, es de interés investigar el efecto del Sorbato de Potasio en la división celular de material biológico sensible como el tejido meristemático de los ápices de las raicillas de Allium cepa L., el cual por su simplicidad de manejo, el gran tamaño de sus células y por la respuesta de su material genético a la presencia de citotóxicos potenciales es usado en el estudio de la dinámica celular, constituyendo un sistema in vitro ideal para estudiar el efecto de los productos químicos que puedan afectar el ciclo celular [10]. Por eso se utiliza como biomonitor para determinar los diferentes efectos ocasionados por químicos [11][12][13].

Cuando las células del tejido meristematico de $A$. cepa $L$. se encuentran en equilibrio proliferativo, la duración de cada uno de los períodos del ciclo celular permanece constante; de esta forma, el número de células que están en una fase determinada es también constante y proporcional a la duración - en tiempo - de la misma; pero cuando se lleva a cabo en presencia de sustancias tóxicas, la división celular de los meristemos radiculares puede inhibirse, ya sea retardando el proceso de mitosis o destruyendo las células ${ }^{15}$. Este tipo de alteraciones generalmente impide el crecimiento normal de la raíz y por tanto, su elongación [14].

El ciclo celular es regulado por la interacción del complejo ciclina - kinasa dependiente de ciclina (CDK) que implica además procesos de transcripción y formación de proteínas que intervienen directamente como elementos regulatorios o interactúan con otros agentes medio ambientales para regular cada uno de los eventos que forman parte del proceso en su conjunto.

La presencia de sustancias antimitóticas, citotóxicas y genotóxicas pueden inducir alteraciones cromosómicas que son el resultado de deficiencias en los mecanismos de control celular y de reparación en el ADN, que se pueden expresar a lo largo del ciclo celular y provocar una gran variedad de efectos sobre el material genético de las células expuestas a agentes físico o químicos; por lo que, se considera que el tiempo de exposición es un factor importante en la inducción de aberraciones cromosómicas. Si las alteraciones no afectan la vida de los organismos, es posible que los individuos que se encuentran expuestas a tales agentes por motivos de trabajo u ocupación, puedan presentar enfermedades genéticas, alteraciones en sus células germinales con el riesgo de ser transmitidas a la descendencia [13][15][16][17]. 
A todo esto, al ensayar en $A$. cepa $L$. el efecto de algunos agentes químicos y físicos, que pueden causar daño a nivel de su ciclo celular, se podría colegir que dichos agentes tendrían el potencial de generar un efecto tóxico en los procesos de división celular en células eucariotas de otros individuos, debido a la universalidad del código genético [18].

Por lo tanto, el objetivo del presente estudio fue el de determinar el efecto del Sorbato de Potasio a $0.5 \mathrm{~g} / \mathrm{l}, 1 \mathrm{~g} / \mathrm{l}$ y $2 \mathrm{~g} / \mathrm{l}$ y tiempos de exposición de 6, 7 y 8 horas sobre el índice mitótico, índice de fases y material genético en meristemos radiculares de $A$. cepa $L$. var. Roja Arequipeña.

\section{METODO EXPERIMENTAL}

\section{Selección y preparación del material biológico.}

Factores de exclusión: Solamente se seleccionaron los bulbos en forma de perilla, color rojo, consistencia dura, peso entre 60 y $70 \mathrm{~g}$ y sin magulladuras ni podredumbres.

De dicho material se escogieron aleatoriamente 12 bulbos de cebolla, a los cuales se les separaron las catáfilas secas y se limpiaron cuidadosamente el disco radicular eliminando todas las raicillas secas sin dañar a dicho disco.

\section{Acondicionamiento del material biológico y selección de bulbos [19] y [20].}

Implementación de los sistemas: Los bulbos de A. cepa $L$., se colocaron en vasos descartables de $100 \mathrm{ml}$ con agua potable procurando mantener sumergidos solo el disco radicular; los sistemas fueron permanentemente aireados con una bomba a razón de $10-20 \mathrm{ml} /$ min. El agua fue renovada diariamente (Fig. 1).

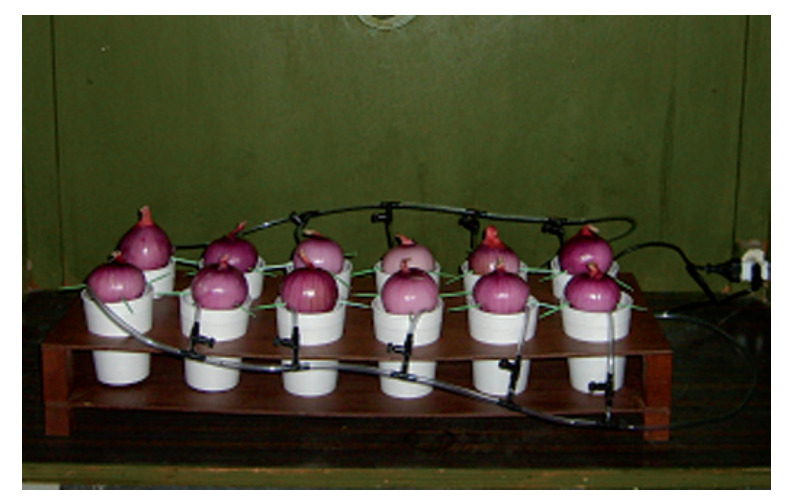

Figura 1. Exposición de las raicillas de Allium cepa L. Var. Roja Arequipeña a las diferentes concentraciones y tiempos de exposición con Sorbato de Potasio.
Selección de bulbos: Después de 72 horas de emergencia de las raicillas, se seleccionaron los bulbos con mayor cantidad de raicillas de longitud promedio de 2 a $3 \mathrm{~cm}$., a fin de asegurar la presencia de células con una cinética mitótica constante.

\section{Diseño experimental para la Exposición de las raicillas [21].}

Los bulbos seleccionados, fueron agruparon por elección al azar para conformar los diferentes tratamientos según concentraciones y tiempos de exposición. El grupo control se mantuvo en agua potable y los otros fueron expuesto a soluciones acuosas de Sorbato de Potasio en concentraciones de 0.5, 1 y $2 \mathrm{~g} / \mathrm{l}$; por 6, 7 y $8 \mathrm{~h}$, el diseño fue totalmente aleatorizado. Se realizaron tres repeticiones por tratamiento, considerando que cada bulbo de cebolla representa a un sistema experimental y que cada raicilla es una unidad experimental (Tabla 1). Los indicadores del efecto ocasionado por el Sorbato de Potasio son: variaciones en los porcentajes de índices mitóticos, de fases y presencia de alteraciones en el material genético; tales efectos fueron comparados con el tratamiento control.

Tabla 1. Diseño experimental para la exposición de las raicillas.

\begin{tabular}{|l|c|c|c|}
\hline Tiempo & $A_{0}$ & $A_{1}$ & $A_{2}$ \\
\hline$B_{0}$ & $A_{0} B_{0}$ & $A_{1} B_{0}$ & $A_{2} B_{0}$ \\
\hline$B_{1}$ & $A_{0} B_{1}$ & $A_{1} B_{1}$ & $A_{2} B_{1}$ \\
\hline$B_{2}$ & $A_{0} B_{2}$ & $A_{1} B_{2}$ & $A_{2} B_{2}$ \\
\hline$B_{3}$ & $A_{0} B_{3}$ & $A_{1} B_{3}$ & $A_{2} B_{3}$ \\
\hline
\end{tabular}

$A_{0}$ : Exposición a 6 horas

$A_{1}$ : Exposición a 7 horas

$\mathbf{B}_{0:}$ Agua potable

$\mathbf{A}_{2}$ : Exposición a 8 horas

$B_{1:}$ Concentración de $0.5 \mathrm{~g} / \mathrm{L}$

$B_{2}$ : Concentración de $1 \mathrm{~g} / \mathrm{L}$

$\mathrm{B}_{3}$ : Concentración de $2 \mathrm{~g} / \mathrm{L}$

\section{Obtención de las raicillas}

De acuerdo al sistema de tiempo exposición creciente después de las seis horas de exposición se seccionaron al azar dos raicillas de cada bulbo, las cuales previamente se lavaron con agua potable, para traspasarla a la solución fijadora de Carnoy's.

Luego los bulbos fueron retornados a cada una de sus respectivas soluciones para realizar el mismo procedimiento a las siete y ocho horas de exposición siguientes. 


\section{Coloración, Obtención y Observación de preparados citológicos [22] y [23].}

Las raíces fueron retiradas de la solución fijadora de Carnoy's, y se lavaron tres veces en agua potable para retirar los restos del fijador. Luego se procedió a secarlas con papel toalla para eliminar los restos de agua de la superficie. Posteriormente fueron depositadas sobre una luna de reloj para su respectiva coloración en Orceína acética al $2 \%$ de acuerdo a la técnica rápida de Tjio y Levan.

Finalmente las células fueron observadas en el microscopio compuesto a 400 y 1000x.

\section{Análisis citológico y evaluación del efecto [22][23]}

Se hizo un recuento de 1500 células meristemáticas de cada uno de los tratamientos, para establecer el índice mitótico y de fases, según las formulas correspondientes y la presencia de figuras mitóticas anormales.

\section{Para determinar el índice mitótico}

IM: Índice Mitótico.

$$
\mathbf{I M}=\frac{\mathbf{N C M}}{\mathbf{N T C}} \times 100
$$

NCM: Número Células en Mitosis.

NTC: Número Total de Células Observadas.

Formula para determinar el índice de fases

IF: Índice de fase.

$$
\text { IF }=\frac{\mathbf{A}}{\mathbf{B}} \times 100
$$

A: Número de células de la fase respectiva.

$B$ : Número total de células en mitosis.
Análisis estadístico [5],[23] y [24]

Se realizó la estimación del análisis de varianza totalmente aleatorizado y la comparación múltiple de promedios, mediante la prueba de Duncan $(p<0.05)$ utilizando el programa estadístico Statgraphics Plus versión 5.1.

\section{RESULTADOS Y DISCUSION}

Las células meristemáticas presentes en los ápices radiculares de Allium cepa $L$. (cebolla), constituyen un modelo ideal para el estudio de los fenómenos celulares y moleculares involucrados en los procesos de división celular, debido a que el 10 al $15 \%$ de células se encuentran en mitosis y a la respuesta de su material genético a la presencia de citotóxicos potenciales en las soluciones de prueba [25]. Al evaluar los Índices Mitóticos (IM), Profásicos (IP), Metafásicos (I Met.), Anafásicos (IA) y Telofásicos (IT) (Fig.7) en células meristemáticas de los tratamientos control, se observa que dichos valores se aproximan a lo reportado por Beltrán y Gonzáles (1995); los cuales estimaron un IM de $14.6 \%$, IP de $44.60 \%$, I Met. de $15.40 \%$, IA de $9.20 \%$ y IT de $28.80 \%$. Las diferencias que existen entre lo reportado en nuestro estudio con el de los autores antes mencionados, probablemente se deberían a diferencias metodológicas; lo cual explicaría la ligera variación en los valores de los índices hallados. [12][26].

Tabla 2. Tratamientos y Promedios de los Índice Mitótico (\%) e Índice de Fases (\%) en

\begin{tabular}{|c|c|c|c|c|c|c|}
\hline \multirow[b]{2}{*}{ TRATAMIENTOS } & \multirow{2}{*}{$\begin{array}{c}\text { INTERFASE } \\
\pm \text { SD }\end{array}$} & \multirow{2}{*}{$\begin{array}{c}\text { MITÓTICO } \pm \\
\text { SD }\end{array}$} & \multicolumn{4}{|c|}{ FASES } \\
\hline & & & $\begin{array}{c}\text { PROFASE } \pm \\
\text { SD }\end{array}$ & $\begin{array}{c}\text { METAFASE } \\
\pm \text { SD }\end{array}$ & $\begin{array}{c}\text { ANAFASE } \pm \\
\text { SD }\end{array}$ & $\begin{array}{c}\text { TELOFASE } \\
\pm \text { SD }\end{array}$ \\
\hline $\mathrm{T}_{1}: 0 \mathrm{~g} / \mathrm{L}-6 \mathrm{~h}$ & $85.54 \pm 0.057$ & $14.46 \pm 0.056$ & $48.70 \pm 0.036$ & $14.74 \pm 0.050$ & $10.06 \pm 0.072$ & $26.50 \pm 0.072$ \\
\hline$T_{2}: 0.5 \mathrm{~g} / \mathrm{L}-6 \mathrm{~h}$ & $86.03 \pm 0.068$ & $13.97 \pm 0.068$ & $53.01 \pm 0.031$ & $13.23 \pm 0.076$ & $9.60 \pm 0.045$ & $24.07 \pm 0.071$ \\
\hline$T_{3}: 1 \mathrm{~g} / \mathrm{L}-6 \mathrm{~h}$ & $86.51 \pm 0.021$ & $13.49 \pm 0.021$ & $65.03 \pm 0.105$ & $11.77 \pm 0.098$ & $8.58 \pm 0.041$ & $14.62 \pm 0.085$ \\
\hline$T_{4}: 2 \mathrm{~g} / \mathrm{L}-6 \mathrm{~h}$ & $88.21 \pm 0.038$ & $11.79 \pm 0.038$ & $69.75 \pm 0.205$ & $9.81 \pm 0.104$ & $7.92 \pm 0.021$ & $12.52 \pm 0.042$ \\
\hline $\mathrm{T}_{5}: 0 \mathrm{~g} / \mathrm{L}-7 \mathrm{~h}$ & $85.44 \pm 0.040$ & $14.56 \pm 0.040$ & $48.78 \pm 0.021$ & $14.70 \pm 0.067$ & $9.98 \pm 0.032$ & $26.53 \pm 0.023$ \\
\hline $\mathrm{T}_{6}: 0.5 \mathrm{~g} / \mathrm{L}-7 \mathrm{~h}$ & $86.54 \pm 0.040$ & $13.46 \pm 0.040$ & $58.66 \pm 0.110$ & $11.79 \pm 0.093$ & $9.53 \pm 0.104$ & $20.03 \pm 0.095$ \\
\hline$T_{7}: 1 \mathrm{~g} / \mathrm{L}-7 \mathrm{~h}$ & $87.95 \pm 0.101$ & $12.05 \pm 0.070$ & $84.97 \pm 0.173$ & $5.82 \pm 0.083$ & $3.53 \pm 0.070$ & $7.23 \pm 0.104$ \\
\hline$T_{8}: 2 \mathrm{~g} / \mathrm{L}-7 \mathrm{~h}$ & $88.43 \pm 0.070$ & $11.57 \pm 0.070$ & $87.40 \pm 0.076$ & $4.49 \pm 0.091$ & $2.59 \pm 0.058$ & $5.53 \pm 0.106$ \\
\hline $\mathrm{T}_{9}: 0 \mathrm{~g} / \mathrm{L}-8 \mathrm{~h}$ & $85.42 \pm 0.040$ & $14.58 \pm 0.040$ & $48.81 \pm 0.025$ & $14.69 \pm 0.011$ & $9.99 \pm 0.025$ & $26.51 \pm 0.026$ \\
\hline$T_{10}: 0.5 \mathrm{~g} / \mathrm{L}-8 \mathrm{~h}$ & $86.93 \pm 0.100$ & $13.07 \pm 0.101$ & $65.83 \pm 0.135$ & $10.29 \pm 0.080$ & $9.47 \pm 0.081$ & $14.24 \pm 0.069$ \\
\hline $\mathrm{T}_{11}: 1 \mathrm{~g} / \mathrm{L}-8 \mathrm{~h}$ & $88.21 \pm 0.130$ & $11.79 \pm 0.131$ & $94.93 \pm 0.058$ & $2.25 \pm 0.101$ & $1.54 \pm 0.064$ & $1.21 \pm 0.045$ \\
\hline $\mathrm{T}_{12}: 2 \mathrm{~g} / \mathrm{L}-8 \mathrm{~h}$ & $88.74 \pm 0.049$ & $11.26 \pm 0.129$ & $95.57 \pm 0.044$ & $2.06 \pm 0.078$ & $1.32 \pm 0.076$ & $0.92 \pm 0.031$ \\
\hline
\end{tabular}
meristemos radiculares de Allium cepa $L$. 
Al analizar los resultados obtenidos a partir de los diferentes tratamientos con Sorbato de Potasio para los IM (Tabla 2), se observa que los valores van disminuyendo significativamente conforme se incrementan las concentraciones, hasta alcanzar un valor de $11.26 \%$ a la concentración de $2 \mathrm{~g} / \mathrm{l}$ durante 8 horas de exposición.

Esto explicaría, que el Sorbato de Potasio, estaría actuando a nivel del segundo punto de control en el ciclo celular $\left(G_{2}\right)$, impidiendo la formación de los complejos CdK1-ciclina B; las cuales forman a su vez el Factor Promotor de la Mitosis (FPM), que permite el paso a través de este punto hacia el período $M$, ello traería como consecuencia que las células no transiten normalmente de la fase $G_{2}$ hacia la mitosis; deteniéndose entonces en interfase y consecuentemente disminuyendo el número de células en división [27] [28].

Así mismo, el ciclo celular es un proceso que demanda gasto de energía a partir de ATP, la cual se realiza durante el intervalo $G_{1}$ caracterizado por la reanudación de la síntesis de ARN y proteínas, que estaba interrumpida durante la mitosis, la fase $S$ donde la célula sintetiza ADN, donde se realiza un gran gasto de energía: por ello la célula entra nuevamente en un proceso de crecimiento y adquisición de ATP para pasar a la fase $G_{2}$ La energía adquirida durante esta fase se utiliza para el proceso de mitosis [29]. En tal sentido, es posible que el Sorbato de Potasio, altere la síntesis de ATP y por lo tanto ocasione una disminución del índice mitótico.

Al analizar los resultados obtenidos a partir de los diferentes tratamientos con Sorbato de Potasio para los IP (Tabla 2) se observa que los valores incrementan significativamente conforme aumentan las concentraciones, hasta alcanzar el $95.57 \%$ a la concentración de $2 \mathrm{~g} / \mathrm{l}$ durante 8 horas de exposición.

El incremento del IP en los tratamientos expuestos al Sorbato de potasio, se debería probablemente a que dicha sustancia al inactivar la formación de los complejos CdK1 - Ciclina B; traería como consecuencia que los procesos de condensación del material genético y de polimerización de microtúbulos para la formación del huso se vean afectados; por lo que las células no transitarían normalmente desde profase hacia metafase. [15][30][31].

Al analizar los resultados obtenidos a partir de los diferentes tratamientos (Tabla 2) para los IME, se observó que los valores van disminuyendo significativamente conforme se incrementan las concentraciones, hasta llegar a $2.06 \%$ la concentración de $2 \mathrm{~g} / \mathrm{l}$ durante $8 \mathrm{~h}$ de exposición.
La disminución del índice metafásico, se debería a que el Sorbato de Potasio al inhibir el complejo Cdk1 - Ciclina B, actúa sobre los centros de organización de microtúbulos, disminuyendo el ensamblaje del huso mitótico. Ello desencadenaría una serie de procesos molecularesquedeterminaríanuncambiosúbito en la funcionalidad de los cinétocoros, dejando de inducir la polimerización de microtúbulos que se elaboran perpendicularmente al eje mayor del cromosoma y se anclan por una de sus extremidades en el cinétocoro, logrando solo pasar a metafase un número reducido de células [29][32].

Los resultados obtenidos a partir de los diferentes tratamientos (Tabla 2) para IA se muestran que los valores van disminuyendo significativamente conforme se incrementan las concentraciones, hasta llegar a $1.32 \%$ a la concentración de $2 \mathrm{~g} / \mathrm{l}$ durante $8 \mathrm{~h}$ de exposición.

La disminución en el IA, estaría ocurriendo debido a que el aumento del número de células en profase generó una disminución en metafase y por ende una disminución en anafase. Por otro lado, el Sorbato de Potasio actuaría inhibiendo la actividad catalítica de las cohesinas, MAPs y catastrofinas; las cuales a su vez se encargan de llevar a cabo el proceso de segregación de cromátidas hermanas; no permitiendo entonces, que las células transiten normalmente hacia anafase y hacia telofase [29][33].

Respecto a la presencia de puentes anafásicos (Figura 2), se observa una mayor cantidad en la concentración de $0.5 \mathrm{~g} / \mathrm{l}$ expuesta a $6 \mathrm{~h}$, y van disminuyendo significativamente conforme aumentan las concentraciones y tiempos ensayados (Tabla 3), esto debería a que el sorbato de potasio ocasiona roturas cromosómicas en los extremos que comprenden el ADN telomérico; lo que posteriormente conduciría a la unión de cromosomas fragmentados (Figura 3 ) durante la anafase-telofase y como consecuencia producirían células hijas con desbalance cromosómico y la posible pérdida de los telómeros por no tener centrómeros [34][35] [36].

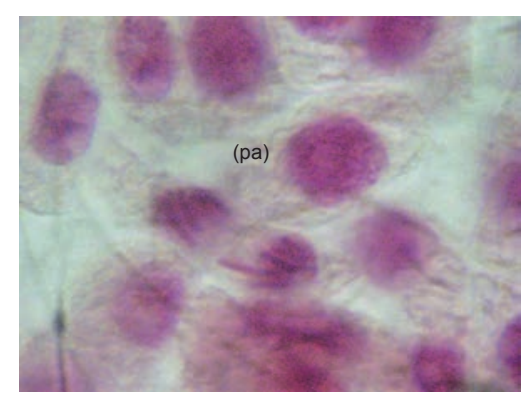

Figura 2. Puente Anafásico (pa) en células meristemáticas de Allium cepa L., a $1 \mathrm{~g} / \mathrm{L}$ de Sorbato de potasio y 6 horas de exposición (1000x). 


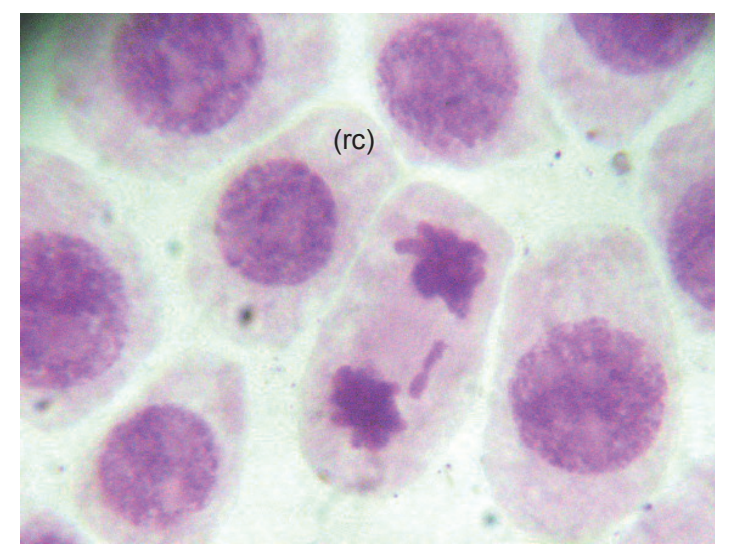

Figura 3. Rupturas ctomosómicas (rc) en meristemos radiculares de Allium cepa, a $2 \mathrm{~g} / \mathrm{L}$ de Sorbato de Potasio y 6 horas de exposición (1000x).

Los resultados obtenidos a partir de los diferentes tratamientos (Tabla 2) para IT se observa que los valores van disminuyendo significativamente conforme se incrementan las concentraciones hasta alcanzar el $0.92 \%$ a la concentración de $2 \mathrm{~g} / \mathrm{L}$ durante $8 \mathrm{~h}$ de exposición.

La disminución del IT, se originaría por el detenimiento del proceso en profase con el consecuente incremento de valores del IP, discutido anteriormente. Sin embargo, debido a que un número reducido de células transitan hasta anafase; solo pasarían hacía la telofase un número reducido de células; lo que explicaría el bajo índice telofásico hallado [37][38].
También se observaron células binucleadas (Figura 4), encontrándose más alto promedio en la concentración de $2 \mathrm{~g} / \mathrm{L}$ durante 8 horas de exposición (Tabla 3 ), y van aumentando significativamente conforme aumentan las concentraciones y tiempos ensayados; esto puede ser debido a que el Sorbato de Potasio originaría una inhibición de la fusión de vesículas del Aparato de Golgi que van desde el centro hacia la periferia de la célula y que a su vez se asocian con microtúbulos entrelazados residuales del huso mitótico, obstaculizando la formación del fragmoplasto responsable de la nueva pared celular durante la telofase [39].

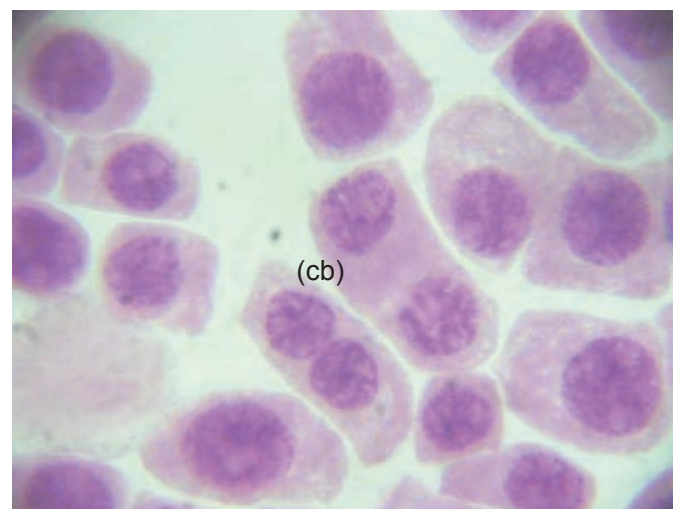

Figura 4. Células binucleadas (cb) en meristemos radiculares en Allium cepa $L$., a $2 \mathrm{~g} / \mathrm{L}$ de Sorbato de potasio y 7 horas de exposición (1000x

Tabla 3. Tratamientos y Promedios de alteraciones en el material genético (\%) en meristemos radiculares de Allium cepa $L$.

\begin{tabular}{|c|c|c|c|}
\hline Tratamientos & $\begin{array}{l}\text { Rupturas } \\
\text { cromosomica } \\
\text { s (\%) } \pm \text { SD }\end{array}$ & $\begin{array}{l}\text { Puentes } \\
\text { Anafásicos } \\
(\%) \pm \text { SD }\end{array}$ & $\begin{array}{l}\text { Células } \\
\text { Binucleadas } \\
(\%) \pm \text { SD }\end{array}$ \\
\hline$T_{1}: 0 \mathrm{~g} / \mathrm{l}-6 \mathrm{~h}$ & $0 \pm 0.0$ & $0 \pm 0.0$ & $0 \pm 0.0$ \\
\hline$T_{2}: 0.5 \mathrm{~g} / \mathrm{l}-6 \mathrm{~h}$ & $0.25 \pm 0.04$ & $0.31 \pm 0.03$ & $0.25 \pm 0.0$ \\
\hline$T_{3}: 1 \mathrm{~g} / \mathrm{l}-6 \mathrm{~h}$ & $0.27 \pm 0.0$ & $0.22 \pm 0.04$ & $0.29 \pm 0.03$ \\
\hline$T_{4}: 2 \mathrm{~g} / \mathrm{l}-6 \mathrm{~h}$ & $0.35 \pm 0.04$ & $0.18 \pm 0.04$ & $0.35 \pm 0.04$ \\
\hline$T_{5}: 0 \mathrm{~g} / \mathrm{l}-7 \mathrm{~h}$ & $0 \pm 0.0$ & $0 \pm 0.0$ & $0 \pm 0.0$ \\
\hline$T_{6}: 0.5 \mathrm{~g} / \mathrm{l}-7 \mathrm{~h}$ & $0.15 \pm 0.04$ & $0.11 \pm 0.04$ & $0.38 \pm 0.04$ \\
\hline$T_{7}: 1 \mathrm{~g} / \mathrm{l}-7 \mathrm{~h}$ & $0.18 \pm 0.04$ & $0.06 \pm 0.0$ & $0.40 \pm 0.0$ \\
\hline$T_{8}: 2 g / l-7 h$ & $0.25 \pm 0.04$ & $0.04 \pm 0.03$ & $0.55 \pm 0.04$ \\
\hline$T_{9}: 0 g / l-8 h$ & $0 \pm 0.0$ & $0 \pm 0.0$ & $0 \pm 0.0$ \\
\hline$T_{10}: 0.5 \mathrm{~g} / \mathrm{l}-8 \mathrm{~h}$ & $0.11 \pm 0.04$ & $0.08 \pm 0.04$ & $0.51 \pm 0.03$ \\
\hline$T_{11}: 1 \mathrm{~g} / \mathrm{l}-8 \mathrm{~h}$ & $0.04 \pm 0.03$ & $0 \pm 0.0$ & $0.54 \pm 0.03$ \\
\hline$T_{12}: 2 g / l-8 h$ & $0.02 \pm 0.03$ & $0 \pm 0.0$ & $0.58 \pm 0.04$ \\
\hline
\end{tabular}




\section{CONCLUSIONES}

El Sorbato de Potasio disminuye el índice mitótico y asimismo aumenta el índice profásico y disminuye los índices metafásico, anafásico y telofásico en células meristemáticas de $A$. cepa $L$. conforme se incrementa la concentración y tiempo de exposición.

El Sorbato de Potasio altera el material genético en células meristemáticas de $A$. cepa L. ocasionando, unión de cromosomas fragmentados, puentes anafásicos y formación de células binucleadas.

\section{AGRADECIMIENTO}

En general quisiera agradecer a todas y cada una de las personas que han compartido conmigo la realización de este trabajo de investigación, y que no necesito nombrar porque tanto ellas como yo sabemos que desde los más profundo de mi corazón les agradezco el haberme brindado todo el apoyo, colaboración, ánimo y sobre todo cariño y amistad.

\section{REFERENCIAS}

[1] Noboa, Miguel.; Evaluación de tortillas de papa refrigerada, bajo el efecto de Sorbato de Potasio como conservante y su influencia en la vida útil (Tesis Pregrado); Universidad Nacional de Chimborazo; Ecuador, 2005.

[2] Sofos, J.N.; Sorbate Food Preservatives; CRC Press, Inc.; Boca Raton; USA; 1989.

[3] Valle Vega, Pedro; Lucas Florentino, Bernardo; Toxicología de Alimentos; México. Rev. del Instituto Nacional de Salud Publica; 2000; 10 (4). 10-12p.

[4] Millán Trujillo, Félix y colaboradores. Estudio de la estabilidad microbiológica del melón (Cucumis melo L) mínimamente procesado por impregnación al vacío, Órgano Oficial de la Sociedad Latinoamericana de Nutrición de Venezuela. 2001. 51(2).

[5] Spiegel, M. R.; Teoría y Problema de Probabilidades y Estadísticas; Serie de compendios Shawn; Edit.Mc Graw Hill; México; 1982; 372p.

[6] Carrandi, S. L.; Efecto de conservantes en la estabilidad de jugo de tuna pasteurizado; Tesis (Ing Agr); Universidad de Chile; Chile; 1995.
[7] Industrias Ragar; México; 2000 - 2008; Disponible en URL:http:// www.quiminet com.mx.

[8] Gerschenson, Lía; Varela, Oscar; Binstock, Guillermo; Food Research Internacional; Rev. Centro de Investigaciones Toxicológicas (Ceitox) de Citefa/Conicet; Facultad de Ciencias Exactas y Naturales; UBA; 2007.

[9] Olaf, C.; Murray, R.; Polenta, G.; D. Lucangeli, C.; Uso de sorbato de potasio y de metil-parabeno para mejorar la conservación de maíz dulce; Rev. ITEA-Producción vegetal; 2002; 98 (2). 127-138 $p$.

[10] Beltrán Orbegoso, R.; Gonzáles Llontop L.; Inducción de puentes cromosómicos permanentes en meristemos de Allium cepa; REBIOL; 1995; 15 (1y 2):9-17p.

[11] Beltran, R.; Gonzales, L. Efecto citotóxico del herbicida 2,4 Diclorofenoxiacetico sobre meristemos de Allium cepa y Vicia Faba; Rebiol; 1997; 17(1 y 2): 15 - 21p.

[12] Gonzáles, C. J.; Efecto antirreparador de la cafeína sobre el daño cromosómico inducido por 2,4 -D sal amina en meristemos de Allium cepa L.; Tesis de bachiller en Ciencias Biológicas; Universidad Nacional de Trujillo, Perú.; 1994.

[13] Otiniano García, M.; Tuesta Collantes, L.; Robles Castillo, H.; Luján Velásquez, M.; Chávez Castillo, M.; Biorremediación de cromo VI de aguas residuales de curtiembres por Pseudomonas sp y su efecto sobre el ciclo celular de Allium cepa, Rev. Medica Vallejiana; 2007; 4 (1).

[14] Díaz, B. M.; Ronco, A.; Pica, G. Y.; Ensayo de toxicidad aguda con Allium cepa L. mediante la evaluación de la inhibición del crecimiento promedio de raíces de cebolla; Rev. Med. Vallejiana; Perú; 2007; 4 (1).

[15] Marcano, L.; Carruyo, I.; Montiel, X.; Bracho, M.; Soto M., L.; Valoración del efecto toxico del cadmio en células meristemáticas de cebolla Allium cepa $L$.; Rev. Fac. Agron. (LUZ); 1999; 16: 476 $487 \mathrm{p}$.

[16] Hernán Barrera, C.; Pardo, L.; Cortina, G.;Evaluación del efecto genotóxico del Antracol WP 70 en cultivos de linfocitos humanos; Revista Médica Del Valle de Colombia: Colombia; 2007; 5 (6). 3-9p. 
[17] Domínguez Odio, A.; Rojas Vázquez, E.; Romero García, L.; Rodríguez Tito; Pérez Andrés, I.; Lesiones genéticas y citológicos inducidas por la exposición a químicos en centros de trabajo de Uruguay, Rev. Salud de los Trabajadores; Uruguay; 2006;14 (1).

[18] Silva, J.; Erdtmann B.; Henriques, J.; Genética toxicológica; Porto Alegre; Primera edición; Editorial Alcance; 2003; $422 \mathrm{p}$.

[19] Andrioli, N.; Wulff, A.; Mudry, M.; Allium cepa como biomonitor de toxicidad y genotoxicidad de metronidazol; Theoría; 2006; 15: 9 - 16p.

[20] Rank, J.; Hviid, M.; A modified Allium test as a tool in the screening of the genotoxicity of complex mixtures; Hereditas; 1993; 1118: 49 - 53p.

[21] Iganci, J. R.V.; Bobrowski, V.I.; G. Heiden, V. C.; Stein, B. H. G. Efecto do extracto aquoso de diferentes especies de Bolbo sobre a germinacao a indice mitótico de Allium cepa L. 2004.

[22] Tijo, J.; Levan, A.; The use of Oxiquinoline in chromosme analysis; Analysis. Ann. Estac. Exptl; Aula Dei; 1950; 2:21-64p.

[23] Beltrán Orbegoso, R. Propuesta de un esquema básico para establecer el perfil genocontaminante de los productos químicos de uso cotidiano mediante el bioindicador Vicia faba $L$. "haba"; SCIENDO; 1999; 2(1-2): 47-55p.

[24] Steel, R.; Torrie, J.; Bioestadística Principios y Procedimientos; $2 \mathrm{~d}^{\mathrm{a}}$ Ed.Edit Mc Graw-Hill S.A; México; 1993.

[25] Vesna, S.; Stegnar, P.; Locka, M.; Toman, M. J.; The evaluation of waste, surfase and ground wáter quality using the Allium test procedure; Mutat. Res.; 1996; 368, $171-179 p$.

[26] Valladolid, A.; Blas, R.; Gonzales, $\mathrm{R}$.; Inducción al recuento de cromosomas somáticos en raíces andinas; Centro Internacional de la Papa (CIP); Perú; 2000.

[27] Shimotohno, A.; Matsubayashi, S.; Yamaguchi, M.; Uchimya, H.; Umeda. Differential phosphorylation activities of CDK - activating Kinases in Arabidopsis thaliana. FEBS Lett. 534. 2003.

[28] Ito, M.; Factors controlling cyclin B expresion .Plant Mol. Biol. 2000. 43: 677$690 p$.
[29] Lodish, H.; Berk, A.; Lawrence, S.; Matsudaira, P.; Baltimore, D.; Darnell, J.; Biología Celular y Molecular; Cuarta Edición; Editorial Medica Panamericana S. A ; . $20 \begin{array}{llll} & 0 & 0 & 2\end{array}$.

[30] Belmont, L. D.; Hyman, A. A.; Sawin, R.E.; Mitchison, T.J.; Real time visualization of cell cycle - dependent changes in microtúbule dinamics in cytoplasmic extracts Cell; 2002, 62.579 $589 p$.

[31] Mitchison, T.J.; Kirshner, M.W.; Dinamic instability of microtúbule growth. Nature; 1999; 312.237- 242p.

[32] Murakami, H.; Nurse, P.; DNA replication and damage checkpoints and meiotic cell cycle controls in the fission and budding yeast; Biochem; 2000; 349:1-12p.

[33] Garcia Herdugo, G.; Fernandez Gómez, M. E.; Hidalgo, J.; López Sáez, J.F.; Effects of protein síntesis inhibition durimng plant mitosis; Cell Res; 2000; 89: 336- 342p.

[34] Castro, R.; Ramírez, V.; Cuenca, P.; Micronúcleos y otras anormalidades nucleares en el epitelio oral de mujeres expuestas ocupacionalmente a plaguicidas; Rev. Biológica tropical; Costa Rica; 2004; 52 (3).

[35] Hernández Fernández, Rolando A. Telómeros y telomerasas, Instituto de Ciencias Básicas y Preclínicas "Victoria de Girón"; Rev Cubana Investigación Biomédica; Cuba; 1999.; 18(2):121-9p.

[36] Prieto, Zulita; León Incio, J.; Quijano Jara, Carlos; Fernández, Radigud; Polo Benites, Edgardo; Vallejo Rodríguez, Roger; Villegas Sánchez, Luís; Efecto Genotóxico del Dicromato de Potasio En Eritrocitos de sangre periférica de Oreochromis niloticus (Tilapia); Rev. Med exp. Salud pública de Perú; 2008; 25(1).

[37] Paniagua, R.; Citologia e Histología Vegetal y Animal; Tercera Edicion; Mc Graw-Hill; Interamericana; 2002.

[38] Murria, A.; Kirschner, M.; Control del ciclo celular; Investigación y Ciencia.;1991; Nro 176

[39] Hawes, C.R.; Brandizzi, F., Andreeva, A.V.; Endomembranes and vesicle trafficking; Current opinion in plant biology; 1999; 2:254-461.

E-mail: yoselyn162002@hotmail.com. 